Article

\title{
Attenuation Zones of Two-Dimensional Periodic Foundations Including the Effect of Vertical Loads
}

\author{
Xinnan Liu ${ }^{1}$, Yihe Wang ${ }^{2}$ and Yingchun Chen ${ }^{3, *}$ \\ 1 School of Civil Engineering, North China University of Technology, Beijing 100144, China; \\ xinnanliu@139.com \\ 2 Beijing Urban Construction Yatai Group Co. Ltd, Beijing 100013, China; wyhat2004@163.com \\ 3 College of Mechanical Engineering and Applied Electronics Technology, Beijing University of Technology, \\ Beijing 100124, China \\ * Correspondence: ychen08089@163.com; Tel.: +86-010-6739-6214
}

Received: 24 January 2019; Accepted: 1 March 2019; Published: 10 March 2019

check for updates

\begin{abstract}
Periodic foundations for seismic isolation are getting increasing attention in the field of civil engineering. Initial stresses due to the vertical loads from the upper structures exist in periodic foundations. In the present paper, attenuation zones of two-dimensional periodic foundations including the effect of vertical loads are studied by the weak form quadrature element method. Comparisons with existing results in special cases without the effect of vertical loads are conducted to validate the proposed method, and good agreement is found. A comprehensive parametric study is conducted to investigate the effects of vertical loads on the attenuation zones. Moreover, frequency-domain and time-domain response analyses of a two-dimensional periodic foundation with finite unit cells are conducted to verify the theoretical results. The results show that bound frequencies of the attenuation zones, corresponding to the vibration mode of the core, are affected by vertical loads. It is also found that vertical loads shift the complete attenuation zones to lower frequencies and reduce the attenuation coefficients in the attenuation zones.
\end{abstract}

Keywords: attenuation zones; periodic foundations; vertical load; mode shape; seismic isolation

\section{Introduction}

As a new kind of seismic isolation technique, periodic foundations possess good seismic isolation performance due to the unique dynamic characteristic of attenuation zones (AZs) [1-5]. Seismic waves with frequencies in the AZs will be attenuated effectively in the periodic foundations [6,7]. Based on the generation mechanism of AZs, periodic foundations can be divided into Bragg scattering periodic foundations and locally-resonant periodic foundations [8-10]. The former enjoy AZs where the corresponding wavelength is of the same order of the geometric size of the unit cells of the periodic foundations. The latter possess AZs with the sizes of unit cells two-orders smaller than the relevant wavelength, providing a possibility to isolate seismic waves with dominant frequencies lower than $20 \mathrm{~Hz}[11,12]$.

The two-dimensional (2D) periodic foundation, which is composed of periodic arrays of heavy core cylinders coated with soft material in stiff matrix, is a typical locally-resonant periodic foundation. Jia and Shi [13] firstly proposed the application of 2D locally-resonant periodic foundations in seismic isolation and systematically the effect of geometric and material parameters on the AZs by using the finite element method. According to the numerical simulation of seismic responses of six-story reinforced concrete frame structures, with a 2D periodic foundation and aseismic rubber bearings, in [13], it is found that the 2D periodic foundation can reduce the seismic response more effectively than the aseismic rubber bearings. Using the finite element method, Cheng and Shi [14] investigated the 
effect of the reinforcing ratio in a concrete matrix, in which it is shown that the effect of the reinforcing ratio in a concrete matrix on the AZs is negligible. Thus, a reinforced concrete matrix can be simplified as plain concrete to determine AZs. More recently, Yan et al. [15] conducted a free field test of a 2D periodic foundation that was made of periodic arrays of iron coated with rubber in concrete matrix, where it was found that the 2D periodic foundation can effectively mitigate the dynamic response of the upper structure for vibration excitations within its AZs.

Periodic foundations, as a type of foundation, are required to support vertical loads due to heavy upper structures. According to the previous research in the aseismic rubber bearings, the seismic isolation performance of aseismic rubber bearings can be affected significantly by the vertical loads $[16,17]$. However, the effect of vertical loads on AZs of 2D periodic foundations has not been studied, which will lead to the discrepancy between the designed and actual AZs of 2D periodic foundations.

In this paper, the weak form quadrature element method is applied to study the AZs of 2D periodic foundations, including the effect of vertical loads. It should be pointed out that wave propagation in layered periodic foundations and 2D periodic foundations are different. Longitudinal waves ( $P$ waves) and shear waves ( $S H$ and $S V$ waves) are decoupled in layered periodic foundations. However, $P$ waves and $S V$ waves are coupled in the plane of the cross section of 2D periodic foundations. The remaining sections are organized as follows: The computational methods are given in Section 2. In Section 3, a validation study of the present method is conducted by comparison with existing results in special cases, without the effect of vertical loads. In Section 4, the effects of vertical loads on the range of AZs and the attenuation coefficients are investigated. In addition, dynamic responses of a 2D periodic foundation with finite unit cells are analyzed. Finally, some conclusions are provided in Section 5.

\section{Computational Method}

Figure 1 shows the cross section of a 2D periodic foundation with vertical loads. The foundation was made of periodic arrays of square steel cylinders coated with rubber in a concrete matrix, where the length of the cylinders in the $y$-direction was assumed to be infinite. Hence, wave propagation in the periodic foundation could be reduced to a 2D problem. A typical unit cell can be defined, as shown in Figure 1, where the side length of square cylinder is $l$, the thickness of coating layer is $t_{k}$, and the periodic constant is $a$. According to the periodic structure theory [18], wave propagation in periodic structures can be investigated by the dynamic analyses of a typical unit cell. In the present paper, the materials of the cores, the coating layers, and the matrix were assumed to be linearly elastic and isotropic. Furthermore, for simplicity, the effect of vertical loads from the upper structure was reduced as homogeneous initial stress, $\sigma_{z z}^{0}$, in the 2D periodic foundation.

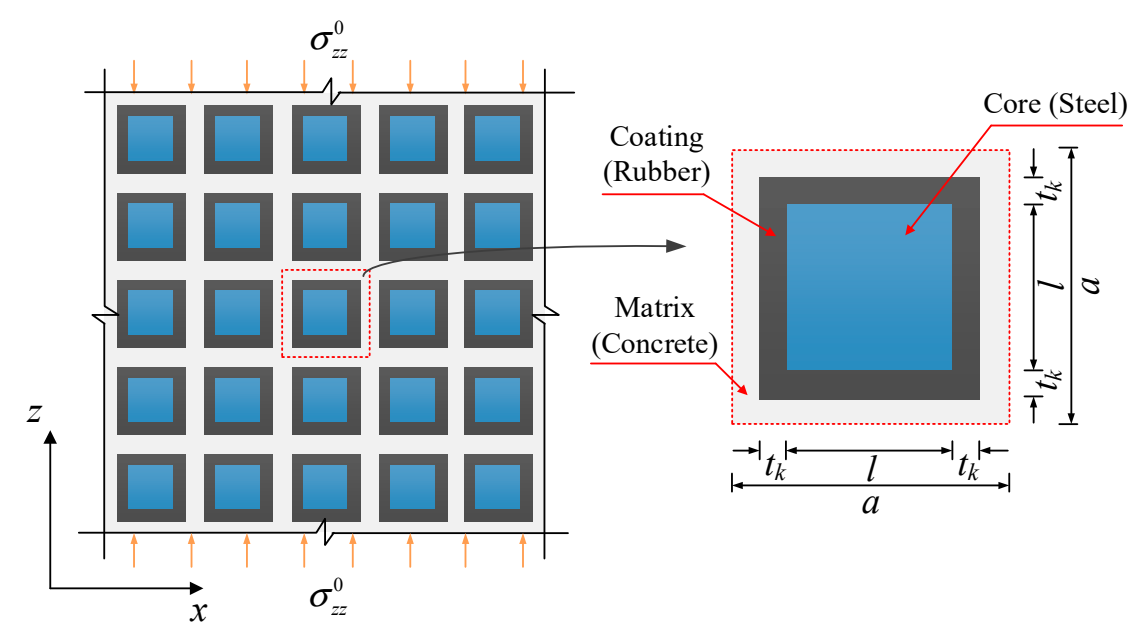

Figure 1. Cross section of a 2D periodic foundation and its typical unit cell. 
According to the weak form quadrature element method, the typical unit cell can be decomposed into several quadrature elements [19]. Let $u$ and $w$ be the displacement components in the $x$ - and $z$-directions, respectively. Thus, the strain energy of the quadrature element $e$ can be expressed as

$$
U^{(e)}=\iint_{\Omega^{(e)}} \frac{1}{2}\left(\mathcal{\varepsilon}^{T} E \mathcal{\varepsilon}+\varepsilon^{* T} \boldsymbol{\sigma}^{0} \mathcal{\varepsilon}^{*}\right) d x d z=\int_{-1}^{1} \int_{-1}^{1}\left(A^{(e)}+A^{0(e)}\right) d \xi d \eta,
$$

where $\Omega^{(e)}$ is the area in the $x-z$ plane of the quadrature element $e, \varepsilon=\left[\begin{array}{lll}\frac{\partial u}{\partial x} & \frac{\partial v}{\partial y} & \frac{\partial v}{\partial x}+\frac{\partial u}{\partial y}\end{array}\right]^{T}$ is the strain vector, $\mathbf{E}=\frac{\bar{E}}{1-\bar{v}^{2}}\left[\begin{array}{ccc}1 & \bar{v} & 0 \\ \bar{v} & 1 & 0 \\ 0 & 0 & (1-\bar{v}) / 2\end{array}\right]$ in which $\left\{\begin{array}{c}\bar{E}=\frac{E}{1-v^{2}} \\ \bar{\mu}=\frac{v}{1-v}\end{array}, E\right.$ is the Young modulus, $v$ is the Poisson ratio, $\varepsilon^{*}=\left[\begin{array}{cc}\frac{\partial u}{\partial z} & \frac{\partial w}{\partial z}\end{array}\right]^{T}, \sigma^{0}=\left[\begin{array}{cc}\sigma_{z z}^{0} & 0 \\ 0 & \sigma_{z z}^{0}\end{array}\right], A^{(e)}$ is the strain energy density of the linear strains, and $A^{0(e)}$ is the strain energy density due to the initial stress terms, respectively.

By using the quadrature and differentiation scheme in the weak form quadrature element method [20-22], Equation (1) can be obtained as

$$
U^{(e)}=\frac{1}{2} \mathbf{d}^{(e)^{T}}\left(\mathbf{K}^{(e)}+\mathbf{K}^{0(e)}\right) \mathbf{d}^{(e)},
$$

where $\mathbf{d}^{(e)}, \mathbf{K}^{(e)}$ and $\mathbf{K}^{0(e)}$ are the nodal displacement vector, stiffness matrix and geometric stiffness matrix of the quadrature element $e$, respectively.

The potential energy of external loads and the kinetic energy of the quadrature element $e$ can be written as

$$
W^{(e)}=-\int_{\partial \Omega^{(e)}} u^{T} \mathbf{f} d s=-\mathbf{d}^{(e) T} \mathbf{F}^{(e)}, T^{(e)}=\frac{1}{2} \dot{\mathbf{d}}^{(e) T} \mathbf{M}^{(e)} \dot{\mathbf{d}}^{(e)},
$$

where $\mathbf{F}^{(e)}$ and $\mathbf{M}^{(e)}$ denote the nodal force vector and the mass matrix of the quadrature element $e$, respectively; the superposed dot denotes derivative with respect to the time.

Applying the Hamilton's principle, $\delta \int_{t_{1}}^{t_{2}} \sum_{e}\left(U^{(e)}+W^{(e)}-T^{(e)}\right) d t=0$, gives the dynamic equations of the typical unit cell

$$
\ddot{\mathbf{M d}}+\left(\mathbf{K}+\mathbf{K}^{0}\right) \mathbf{d}=\mathbf{F},
$$

A harmonic wave with angular frequency $\omega$ and wave vector $\mathbf{k}=\left(k_{x}+\mathrm{i} \varepsilon_{x}\right) \mathbf{e}_{x}+\left(k_{z}+\mathrm{i} \varepsilon_{z}\right) \mathbf{e}_{z}$ is considered, where the real parts $k_{x}$ and $k_{z}$ of the wave vector are phase constants in the $x$ and $z$-directions, respectively; the imaginary parts $\varepsilon_{x}$ and $\varepsilon_{z}$ are attenuation coefficients in the $x$ and $z$-directions, respectively. By applying the Bloch theorem, periodic boundary conditions can be obtained

$$
\begin{gathered}
\mathbf{d}_{r}=\mathrm{e}^{-\varepsilon_{x} a} \mathrm{e}^{\mathrm{i} k_{x} a} \mathbf{d}_{l}, \mathbf{d}_{t}=\mathrm{e}^{-\varepsilon_{z} a} \mathrm{e}^{\mathrm{i} k_{z} a} \mathrm{~d}_{b}, \\
\mathbf{F}_{r}=-\mathrm{e}^{-\varepsilon_{x} a} \mathrm{e}^{\mathrm{i} k_{x} a} \mathbf{F}_{l}, \mathbf{F}_{t}=-\mathrm{e}^{-\varepsilon_{z} a} \mathrm{e}^{\mathrm{i} k_{z} a} \mathbf{F}_{b},
\end{gathered}
$$

where the subscripts $r, l, t$ and $b$ refer to the nodes on the right, left, top and bottom edges of the typical unit cell, respectively. From Equation (5), it can be clearly seen that $\mathrm{e}^{-\varepsilon_{x} a}$ and $\mathrm{e}^{-\varepsilon_{z} a}$ quantify the attenuation of nodal displacement and nodal force between the opposite edges in the $x$ - and $z$-directions, respectively.

Combining Equations (4) and (5) yields

$$
\left[\overline{\mathbf{K}}(\mathbf{k})+\overline{\mathbf{K}}^{0}(\mathbf{k})-\omega^{2}(\mathbf{k}) \overline{\mathbf{M}}\right]\left[\begin{array}{l}
\mathbf{d}_{i} \\
\mathbf{d}_{l} \\
\mathbf{d}_{b}
\end{array}\right]=\left[\begin{array}{l}
0 \\
0 \\
0
\end{array}\right]
$$


where $\overline{\mathbf{K}}(\mathbf{k}), \overline{\mathbf{K}}^{0}(\mathbf{k})$, and $\overline{\mathbf{M}}$ are the corresponding stiffness matrix, geometric stiffness matrix, and mass matrix of the typical unit cell, respectively, after considering the periodic boundary conditions. It should be noted that the mass matrix is independent of the wave vector [23].

The dispersion curves of in-plane waves (coupled $P$ and $S V$ waves) in 2D periodic foundations can be constructed from Equation (6) by finding eigenfrequencies $\omega$ for a given wave vector in the first irreducible Brillouin zone without attenuation coefficients (i.e., $\mathbf{k}=k_{x} \mathbf{e}_{x}+k_{z} \mathbf{e}_{z}$ ). On the other hand, the attenuation coefficients $\varepsilon_{x}$ and $\varepsilon_{z}$ can be determined from Equation (6) for a given angular frequency $\omega$.

\section{Validation of Computational Method}

Two examples were studied to verify the present method by comparison with the available results in special cases without the effect of vertical loads.

\subsection{AZ of 2D Bragg Scattering Periodic Structure}

Using the finite difference method, Liu et al. [24] studied the AZs of a 2D Bragg scattering periodic structure that was made of periodic $\mathrm{Pb}$ inclusions in rubber matrix, which is a special case of the present work by taking the core and the coating layer as the $\mathrm{Pb}$ inclusion. The mass density and elastic coefficients $C_{11}$ and $C_{44}$ of $\mathrm{Pb}$ were $11,600 \mathrm{~kg} / \mathrm{m}^{3}, 72.1 \mathrm{GPa}$, and $14.9 \mathrm{GPa}$, respectively. The mass density and elastic coefficients $C_{11}$ and $C_{44}$ of rubber were $1300 \mathrm{~kg} / \mathrm{m}^{3}, 680 \mathrm{kPa}$, and $40 \mathrm{k} \mathrm{Pa}$, respectively. Figure 2 shows the dispersion curves calculated by the present method and the finite difference method, where the filling ratio of $\mathrm{Pb}$ in the typical unit cell was 0.36 . In Figure 2, the horizontal ordinate represents wave vectors along the boundary of the first irreducible Brillouin zone, and the vertical ordinate represents wave frequencies. Each pair of wave vector and frequency corresponded to a wave propagation mode. For waves with frequencies in the shaded area in Figure 2, there were no corresponding wave propagation mode, which means that these waves were forbidden in the periodic structure. Thus, the frequency range of the shaded area was named as AZ. The solid lines and scatters in Figure 2 represent the results obtained by the present method and the finite difference method, respectively. It can be seen from Figure 2 that the AZs determined by the present method matched well with those by the finite difference method.

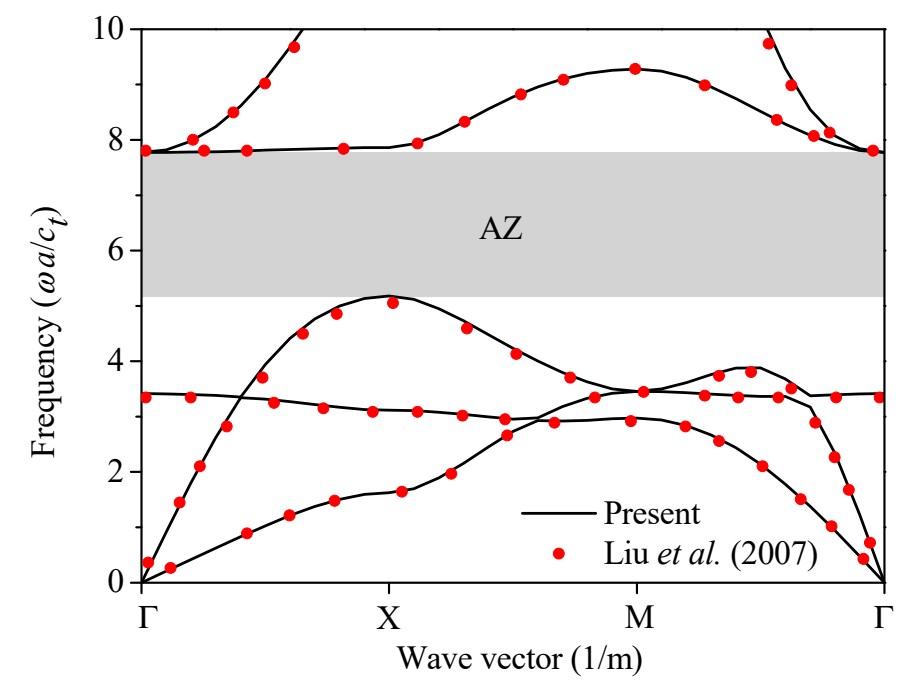

Figure 2. Dispersion curves of in-plane waves in a 2D Bragg scattering periodic structure.

\subsection{AZ of 2D Locally-Resonant Periodic Foundation}

The finite element method (FEM) has been extensively used in the study of periodic structures [25-27]. In the present paper, the matrix, coating layers, and square cores were made of concrete, rubber, and steel, respectively, and whose material parameters are listed in Table 1. We set 
the geometric parameters mentioned in Section 2 as $a=2 \mathrm{~m}, t_{k}=0.2 \mathrm{~m}$ and $l=1 \mathrm{~m}$. Figure 3 shows the dispersion curves of in-plane waves in the 2D locally-resonant periodic foundation, where the continuous lines represent results obtained by the present method and the solid circles represent results given by the FEM. It can be seen from Figure 3 that the AZ ranged from 4.69 to $7.49 \mathrm{~Hz}$. Moreover, agreement in terms of the range of the AZ by the above two methods was good, which validates the present method again.

Table 1. Material parameters.

\begin{tabular}{cccc}
\hline Materials & $\begin{array}{c}\text { Mass Density } \\
\boldsymbol{\rho}\left(\mathbf{k g} / \mathbf{m}^{\mathbf{3}}\right)\end{array}$ & $\begin{array}{c}\text { Young Modulus } \boldsymbol{E} \\
(\mathbf{G P a})\end{array}$ & Poisson Ratio $\boldsymbol{v}$ \\
\hline Steel & 7850 & 210 & 0.330 \\
Rubber & 1300 & $1.37 \times 10^{-4}$ & 0.463 \\
Concrete & 2300 & 25 & 0.330 \\
\hline
\end{tabular}

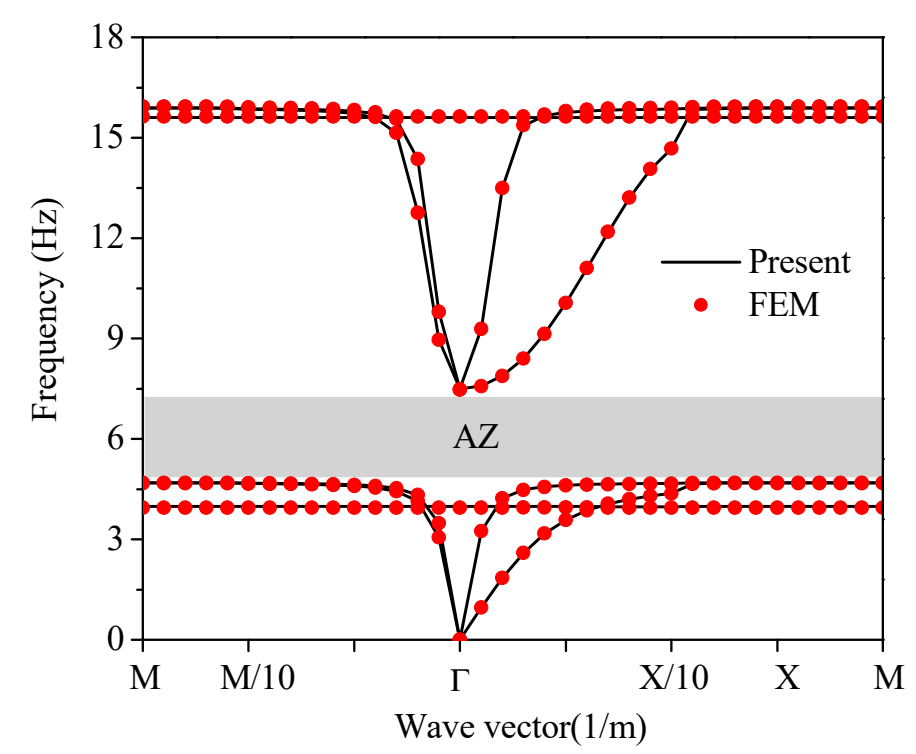

Figure 3. Dispersion curves of in-plane waves in a 2D locally-resonant periodic foundation.

\section{Results and Discussion}

\subsection{Periodic Foundation with Infinite Unit Cells}

Figure 4 shows the dispersion curves in the 2D locally-resonant periodic foundation in Section 3.2, where the initial stress due to the vertical loads was taken as $\sigma_{z z}^{0}=-40 \mathrm{kPa}$. Figure 5 shows the mode shape of frequencies $A_{1}, A_{2}, B_{1}$, and $B_{2}$. Combing Figures $3-5$, it can be found that the unit cell was symmetric in terms of both the geometric and mechanical aspects, without considering the effect of vertical load. Thus, the bound frequencies of the AZ, which were corresponding to the vibration modes of the steel core in the $x$ - and $z$ - directions, were identical. However, after including the effect of vertical loads, the bound frequency corresponding to the vibration modes of the steel core in the $x$-direction decreased more than that in the $z$-direction, which led to the discrepancy between the AZ bounded by $A_{1}$ and $B_{1}$ and that by $A_{2}$ and $B_{2}$, as shown in Figure 4 . For vibration modes of the steel core in the $x$-direction, there were two AZs below $10 \mathrm{~Hz}$, which were $4.24 \sim 6.09$ and 8.44 9.58 Hz, respectively. For vibration modes of the steel core in the $z$-direction, there was only one AZ below $10 \mathrm{~Hz}$, which was $4.37 \sim 6.85 \mathrm{~Hz}$. According to the definition of complete AZ, the complete $\mathrm{AZ}$ with $\sigma_{z z}^{0}=-40 \mathrm{kPa}$ was $4.37 \sim 6.09 \mathrm{~Hz}$, which was lower and narrower than that without vertical load. 


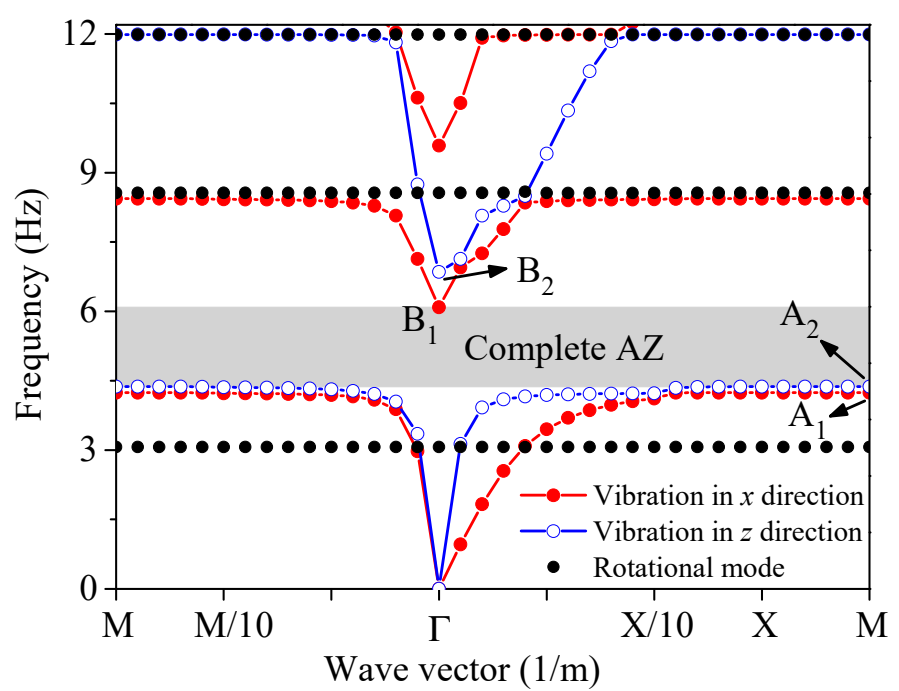

Figure 4. Dispersion curves of in-plane waves in the 2D periodic foundation with $\sigma_{z z}^{0}=-40 \mathrm{kPa}$.

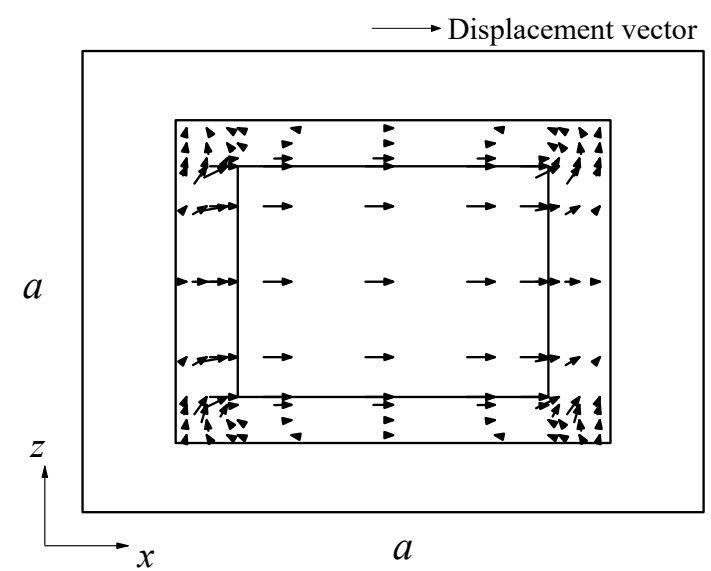

(a) $\mathrm{A}_{1}$

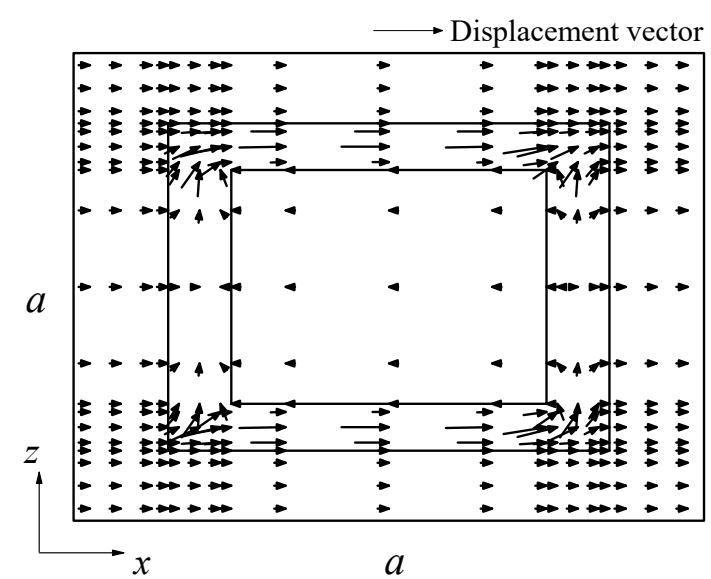

(c) $\mathrm{B}_{1}$

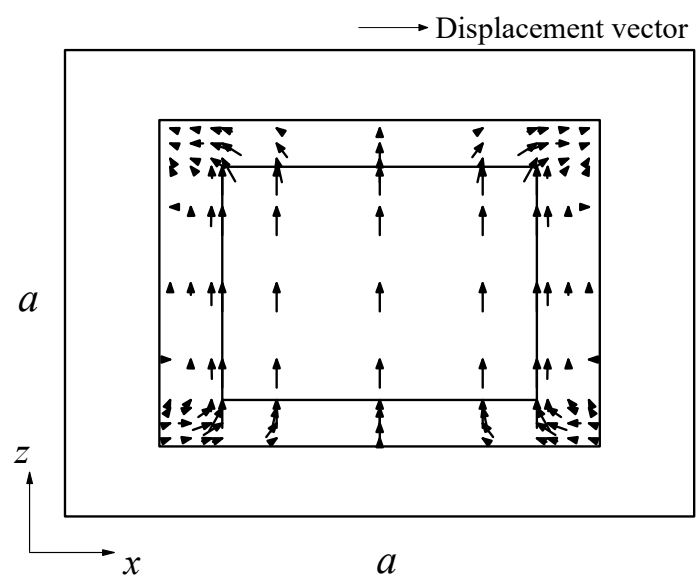

(b) $\mathrm{A}_{2}$

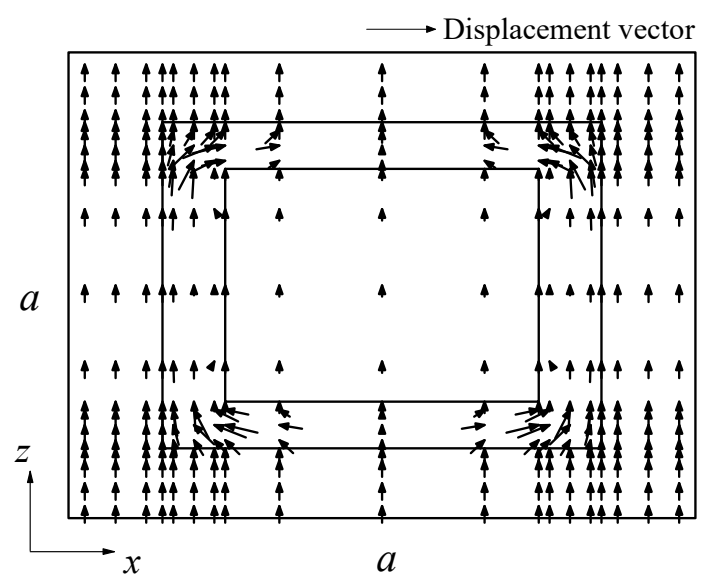

(d) $\mathrm{B}_{2}$

Figure 5. Mode shapes of the unit cell at the bound frequencies at (a) $A_{1},\left(\right.$ b) $A_{2},(\mathbf{c}) B_{1}$ and (d) $B_{2}$.

The width of the complete $A Z$ was defined as $W A Z=U B F-L B F$, where UBF and LBF are the upper- and lower-bound frequencies of the complete $A Z$, respectively. The influence of the initial 
stress on the complete AZ is shown in Figure 6. It could be found that both the LBF and UBF decrease as the compressive initial stress increases. Moreover, the decrease of the UBF was more than that of the LBF, resulting in the decrease of WAZ. The reason is that the stiffness of the unit cell to resist displacement in the $x$-direction was reduced more than that in the $z$-direction. It resulted in the UBF, corresponding to the vibration mode of the steel core in the $x$-direction, being more sensitive to the change of compressive initial stress.

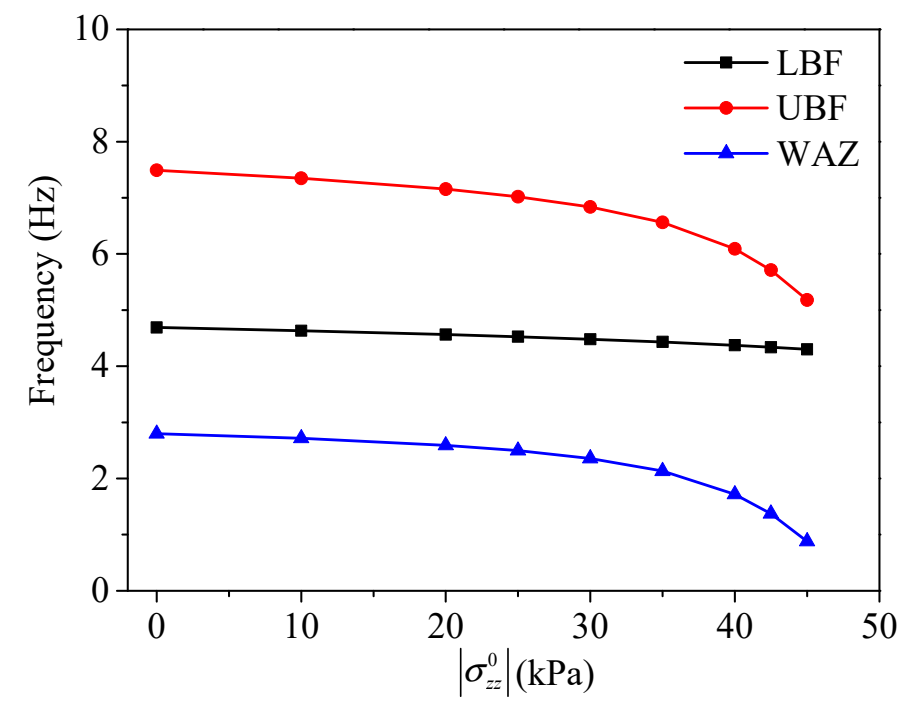

Figure 6. Complete attenuation zone changing with the compressive initial stress.

Figure 7 shows the attenuation coefficients in complete AZs with different initial stresses. It can be seen from Figure $7 \mathrm{a}$ that the complete $\mathrm{AZ}$ became lower and narrower with the increase of the compressive initial stress, which was in accordance with the results in Figure 6. In addition, the attenuation coefficients in the complete $\mathrm{AZ}$ decreased with the increase of the compressive initial stress. From Figure $7 b$, it can be further seen that the compressive initial stress mainly affected the attenuation coefficients below the center frequency of the complete AZ.

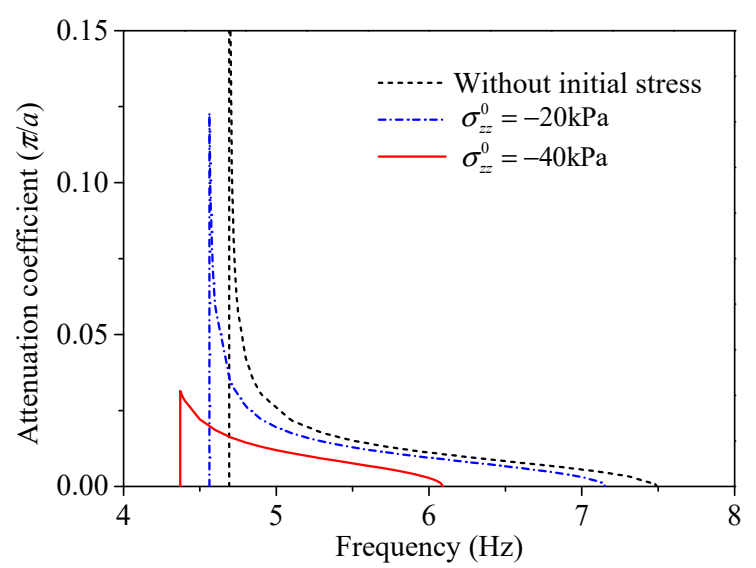

(a)

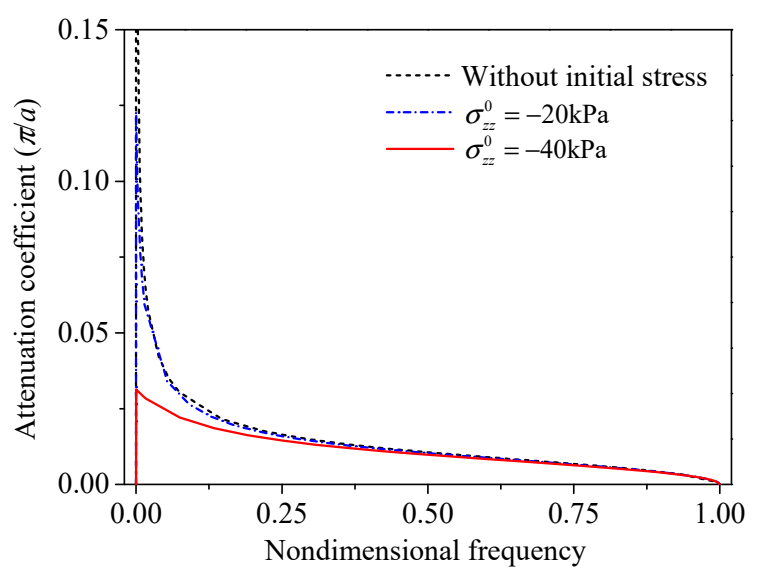

(b)

Figure 7. Attenuation coefficients versus (a) frequency and (b) non-dimensional frequency with different initial stresses.

\subsection{Periodic Foundation with Finite Unit Cells}

In previous sections, attenuation zones, including the effect of vertical load, were studied by assuming that the $2 \mathrm{D}$ periodic foundation consisted of infinite unit cells in both the $x$ - and $z$-directions. 
In the engineering field, the thickness of a foundation is limited, and its length and width are quite larger than its thickness in most cases. Hence, we considered a periodic foundation with five unit cells in the $z$-direction and infinite unit cells in the $x$-direction, as shown in Figure 8. In order to reduce the computational work, periodic boundary conditions, $\mathbf{d}_{r}=\mathbf{d}_{l}$ and $\mathbf{F}_{r}=-\mathbf{F}_{l}$, were applied to the edges of unit cells in the $x$-direction. Displacement excitation was applied to the bottom edge of the five-unit-cell periodic foundation, and the top edge was assumed to be free.

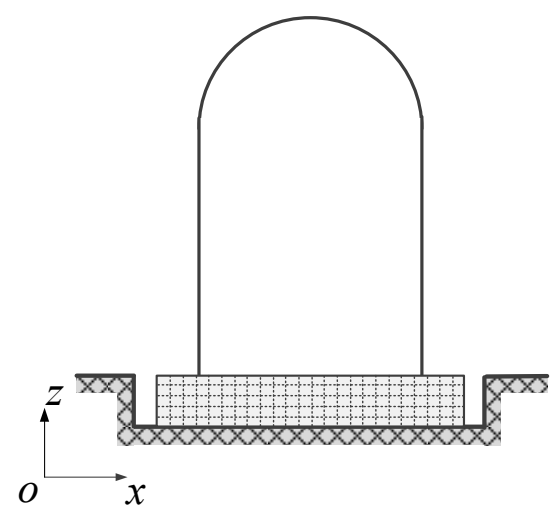

(a)

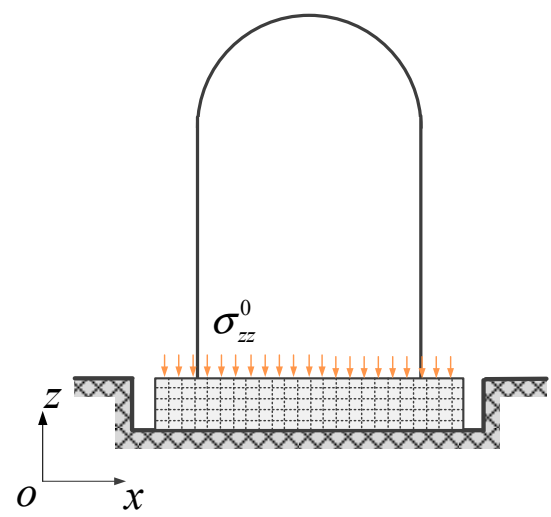

(b)

Figure 8. 2D periodic foundation (a) without and (b) with considering the effect of vertical load.

\subsubsection{Frequency-Domain Dynamic Responses}

The average frequency response function (AFRF) was defined as AFRF $=20 \log _{10}\left(\bar{\delta}_{0} / \delta_{i}\right)$, where $\delta_{i}$ and $\bar{\delta}_{o}$ are the average displacement excitation of the bottom edge and the average displacement response of the top edge of the periodic foundation, respectively. The excitation frequency varied from 0.01 to $12.01 \mathrm{~Hz}$ with a step of $0.05 \mathrm{~Hz}$. Figure 9 shows the AFRFs of the five-unit-cell periodic foundation under $S V$ waves. It should be noted that the steel core moved in the $x$-direction under $S V$ waves. It can be seen from Figure 9 that the frequency ranges with significant attenuation of the excitation agreed very well with the AZs obtained in Figures 3 and 4.

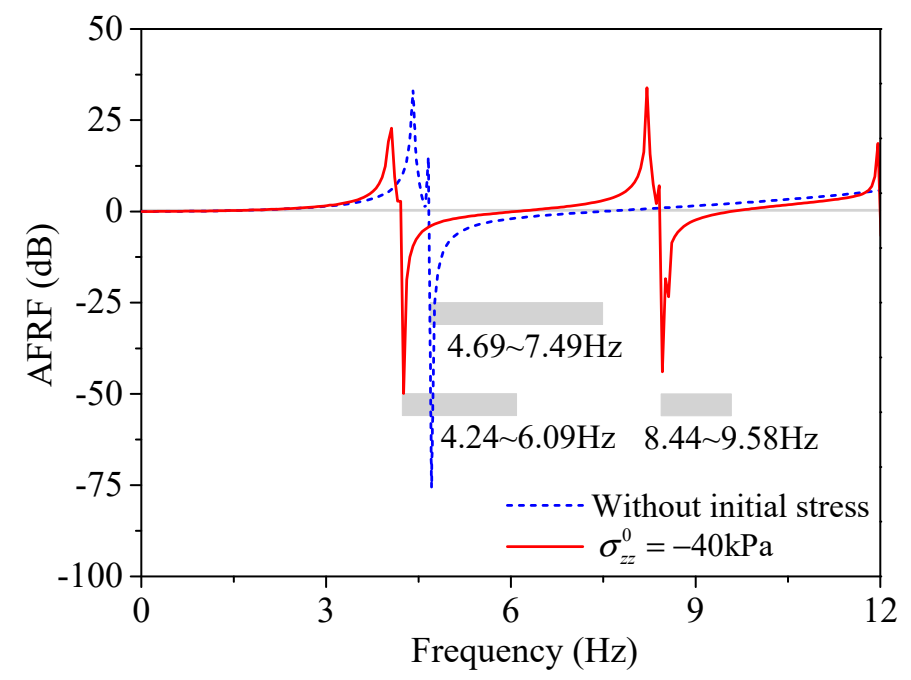

Figure 9. Average frequency response function (AFRFs) of the five-unit-cell periodic foundation under $S V$ waves.

Figure 10 shows the AFRFs of the five-unit-cell periodic foundation under $P$ waves, where the steel core moved in the $z$-direction. It can be seen from Figure 10 that the frequency ranges with significant attenuation were in accordance with the findings in Section 4.1, for both the case with initial 
stress and without initial stress, which again verifies the results for the 2D periodic foundation with infinite unit cells.

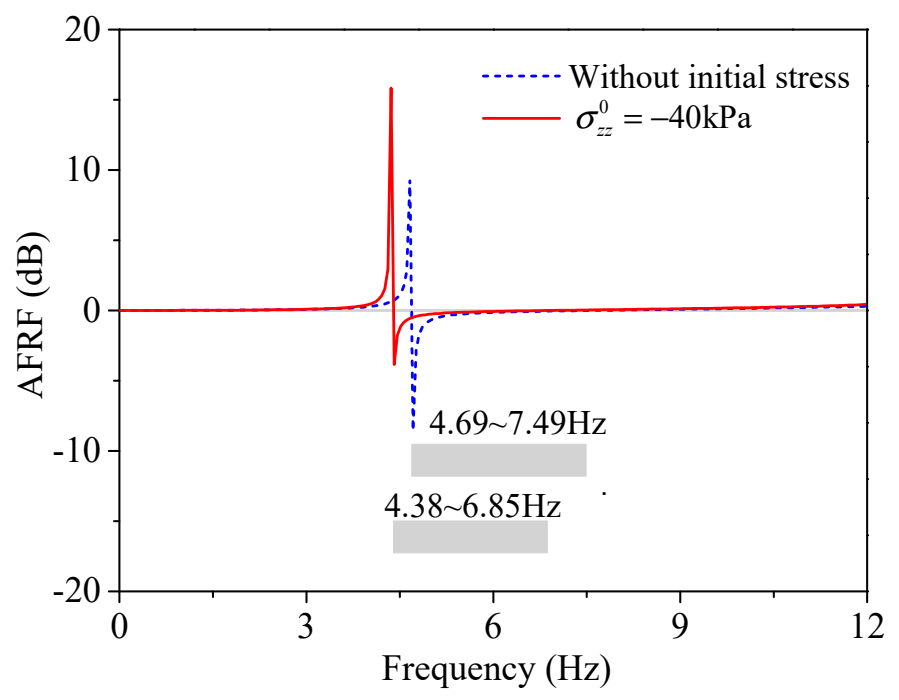

Figure 10. AFRFs of the five-unit-cell periodic foundation under $P$ waves.

\subsubsection{Time-Domain Dynamic Responses}

To further verify the frequency-domain response analyses, a harmonic $S V$ wave $u=\sin (2 \pi f \cdot t)(\mathrm{mm})$ was considered as excitation to the bottom edge of the five-unit-cell periodic foundation, where $f$ is the frequency of the excitation and the time interval is taken as $\Delta t=1 /(80 f)$. Figure 11 shows the averaged dynamic responses of the top edge of the periodic foundation under $S V$ wave, with $f=4.3 \mathrm{~Hz}$. It is noted that the $\mathrm{AZ}$ for $S V$ waves of the periodic foundation without initial stress was $4.69 \sim 7.49 \mathrm{~Hz}$, and those with initial stress $\sigma_{z z}^{0}=-40 \mathrm{kPa}$ were $4.24 \sim 6.09 \mathrm{~Hz}$ and 8.44 9.58 Hz. The excitation frequency $f=4.3 \mathrm{~Hz}$ was inside the AZs with initial stress $\sigma_{z z}^{0}=-40 \mathrm{kPa}$, but outside the $\mathrm{AZ}$ without initial stress. It can be seen from Figure 11 that the averaged dynamic response, of the top edge of the periodic foundation, with initial stress $\sigma_{z z}^{0}=-40 \mathrm{kPa}$ was significantly smaller than that without initial stress.

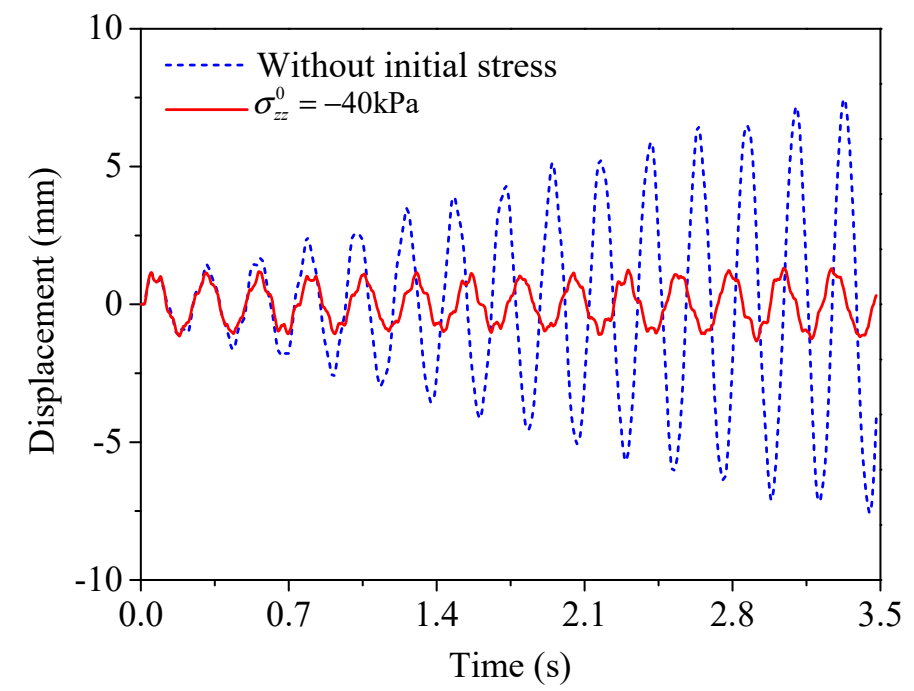

Figure 11. Averaged dynamic responses of the top edge of the periodic foundation under $S V$ wave with $f=4.3 \mathrm{~Hz}$.

Figure 12 shows the averaged dynamic responses of the top edge of the periodic foundation under $S V$ wave with $f=7 \mathrm{~Hz}$. Note that the excitation frequency $f=7 \mathrm{~Hz}$ was inside the AZ without initial 
stress but outside the AZs with initial stress $\sigma_{z z}^{0}=-40 \mathrm{kPa}$. According to the results in Figure $7 \mathrm{a}$, the attenuation coefficient at $f=7 \mathrm{~Hz}$ was quite small in the $\mathrm{AZ}$ without initial stress. It can be seen from Figure 12 that the averaged dynamic response of the top edge of the periodic foundation without initial stress was slightly smaller than that with initial stress $\sigma_{z z}^{0}=-40 \mathrm{kPa}$, which supports both the theoretical analyses and the frequency-domain response analyses.

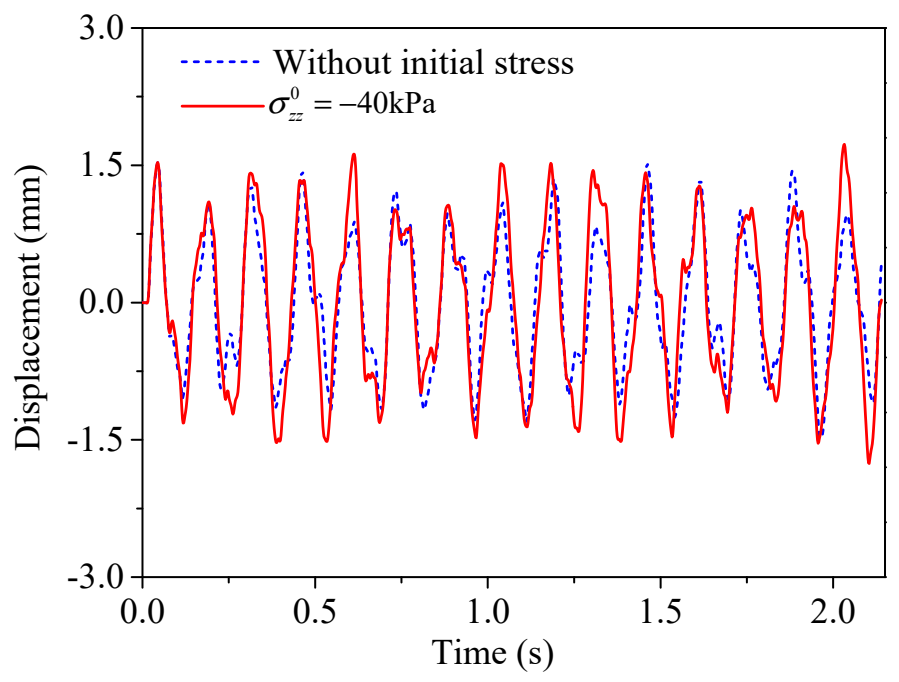

Figure 12. Averaged dynamic responses of the top edge of the periodic foundation under $S V$ wave with $f=7 \mathrm{~Hz}$.

\section{Conclusions}

Combining the weak form quadrature element method and the Bloch theorem, the effect of vertical loads on AZs of 2D locally-resonant periodic foundations were studied by simplifying the effect of vertical loads as initial stresses. The present method was validated with existing results in special cases. By the theoretical analyses of a periodic foundation with infinite unit cells and the dynamic analyses of a periodic foundation with finite unit cells, the following conclusions could be drawn:

(1) For the case without considering the effect of vertical loads, the AZs of 2D locally-resonant periodic foundations for $P$ waves and $S V$ waves are identical. For the case including the effect of vertical loads, AZs for $S V$ waves are more sensitive to the change of vertical loads than those for $P$ waves.

(2) The complete AZ is of lower frequency and its width is narrower when the effect of vertical loads is considered. That implies that the width of the designed complete AZ, without including the effect of vertical loads, is overestimated.

(3) Vertical loads weaken excitation attenuation in the complete AZ, especially for excitation with frequencies below the center of the complete AZ.

It should be noted that one of the limitations of the present study was the reduction of vertical loads into uniformly-distributed initial stresses. Since the effect of vertical loads are mainly localized near the columns in the ground floor, one of the possible improvements of the present study is numerical analysis of the effect of vertical loads in practical engineering application, by using the FEM and the weak form quadrature element method.

Author Contributions: X.L. and Y.C. conceived this original work; Y.W. performed the numerical analysis; X.L. and Y.W. wrote the paper; Y.C. edited and polished the paper.

Funding: This research was funded by the National Natural Science Foundation of China (51808007) and "Rixin Scientist" of Beijing University of Technology.

Acknowledgments: This work is supported by the National Natural Science Foundation of China (51808007) and "Rixin Scientist" of Beijing University of Technology. 
Conflicts of Interest: The authors declare no conflicts of interest.

\section{References}

1. Liu, X.N.; Shi, Z.F.; Mo, Y.L.; Cheng, Z.B. Effect of initial stress on attenuation zones of layered periodic foundations. Eng. Struct. 2016, 121, 75-84. [CrossRef]

2. Kim, S.; Das, M.P. Seismic waveguide of metamaterials. Mod. Phys. Lett. B 2012, 26, 1250105. [CrossRef]

3. Kim, S.; Das, M.P. Artificial seismic shadow zone by acoustic metamaterials. Mod. Phys. Lett. B 2013, 27, 1350140. [CrossRef]

4. Aravantinos-Zafiris, N.; Sigalas, M.M. Large scale phononic metamaterials for seismic isolation. J. Appl. Phys. 2015, 118, 64901. [CrossRef]

5. Cheng, Z.B.; Shi, Z.F. Composite periodic foundation and its application for seismic isolation. Earthq. Eng. Struct. D 2018, 47, 925-944. [CrossRef]

6. Hussein, M.I.; Leamy, M.J.; Ruzzene, M. Dynamics of phononic materials and structures: Historical origins, recent progress and future. Appl. Mech. Rev. 2014, 66, 40802. [CrossRef]

7. Liu, X.N.; Shi, Z.F.; Mo, Y.L. Effect of initial stress on periodic Timoshenko beams resting on an elastic foundation. J. Vib. Control 2017, 23, 3041-3054. [CrossRef]

8. Huang, J.K.; Shi, Z.F.; Huang, W.X.; Chen, X.H.; Zhang, Z.W. A periodic foundation with rotational oscillators for extremely low-frequency seismic isolation: Analysis and experimental verification. Smart Mater. Struct. 2017, 26, 35061. [CrossRef]

9. Huang, J.K.; Liu, X.W.; Chen, X.H.; Xiang, H.J. Multiple flexural-wave attenuation zones of periodic slabs with cross-like holes on an arbitrary oblique lattice: Numerical and experimental investigation. J. Sound Vib. 2018, 437, 135-149. [CrossRef]

10. He, J.H.; Huang, H.H. Tunable acoustic wave propagation through planar auxetic metamaterial. J. Mech. 2018, 34, 113-122. [CrossRef]

11. Xiang, H.J.; Shi, Z.F.; Wang, S.J.; Mo, Y.L. Periodic materials-based vibration attenuation in layered foundations: Experimental validation. Smart Mater. Struct. 2012, 21, 112003. [CrossRef]

12. Miniaci, M.; Krushynska, A.; Bosia, F.; Pugno, N.M. Large scale mechanical metamaterials as seismic shields. New J. Phys. 2016, 18, 83041. [CrossRef]

13. Jia, G.F.; Shi, Z.F. A new seismic isolation system and its feasibility study. Earthq. Eng. Eng. Vib. 2010, 9, 75-82. [CrossRef]

14. Cheng, Z.B.; Shi, Z.F. Novel composite periodic structures with attenuation zones. Eng. Struct. 2013, 56, 1271-1282. [CrossRef]

15. Yan, Y.Q.; Laskar, A.; Cheng, Z.B.; Meng, F.; Tang, Y.; Mo, Y.L.; Shi, Z.F. Seismic isolation of two dimensional periodic foundations. J. Appl. Phys. 2014, 116, 44908. [CrossRef]

16. Ryan, K.L.; Kelly, J.M.; Chopra, A.K. Nonlinear model for lead-rubber bearings including axial-load effects. J. Eng. Mech. 2005, 131, 1270-1278. [CrossRef]

17. Ryan, K.L.; Chopra, A.K. Estimating seismic demands for isolation bearings with building overturning effects. J. Struct. Eng. 2006, 132, 1118-1128. [CrossRef]

18. Peng, H.; Pai, P.F. Acoustic metamaterial plates for elastic wave absorption and structural vibration suppression. Int. J. Mech. Sci. 2014, 89, 350-361. [CrossRef]

19. Zhong, H.Z.; Yu, T. A weak form quadrature element method for plane elasticity problems. Appl. Math. Model. 2009, 33, 3801-3814. [CrossRef]

20. Yuan, S.; Du, J.N. Effective stress-based upper bound limit analysis of unsaturated soils using the weak form quadrature element method. Comput. Geotech. 2018, 98, 172-180. [CrossRef]

21. Yuan, S.; Du, J.N. Upper bound limit analysis using the weak form quadrature element method. Appl. Math. Model. 2018, 56, 551-563. [CrossRef]

22. Liu, X.N.; Shi, Z.F.; Xiang, H.J.; Mo, Y.L. Attenuation zones of periodic pile barriers with initial stress. Soil Dyn. Earthq. Eng. 2015, 77, 381-390. [CrossRef]

23. El-Sabbagh, A.; Akl, W.; A, B. Topology optimization of periodic Mindlin plates. Finite Elem. Anal. Des. 2008, 44, 439-449. [CrossRef]

24. Liu, Y.H.; Chang, C.C.; Chern, R.L.; Chang, C.C. Phononic band gaps of elastic periodic structures: A homogenization theory study. Phys. Rev. B 2007, 75, 54104. [CrossRef] 
25. Langlet, P.; Hladky-Hennion, A.C.; Decarpigny, J.N. Analysis of the propagation of plane acoustic waves in passive periodic materials using the finite element method. J. Acoust. Soc. Am. 1995, 98, 2792-2800. [CrossRef]

26. Khelif, A.; Aoubiza, B.; Mohammadi, S.; Adibi, A.; Laude, V. Complete band gaps in two-dimensional phononic crystal slabs. Phys. Rev. E 2006, 74, 46610. [CrossRef] [PubMed]

27. Gao, N.S.; Wu, J.H.; Yu, L. Research on bandgaps in two-dimensional phononic crystal with two resonators. Ultrasonics 2015, 56, 287-293. [CrossRef] [PubMed]

(c)

(c) 2019 by the authors. Licensee MDPI, Basel, Switzerland. This article is an open access article distributed under the terms and conditions of the Creative Commons Attribution (CC BY) license (http:/ / creativecommons.org/licenses/by/4.0/). 